\title{
Classical and semiclassical non-perturbative treatments of the electron transfer process in the collisional system proton-hydrogen involving initial excited states
}

\author{
A. Taoutioui ${ }^{1,2}$, A. Dubois ${ }^{1}$, N. Sisourat ${ }^{1}$ and A. Makhoute ${ }^{2}$ \\ ${ }^{1}$ Sorbonne Université, CNRS, Laboratoire de Chimie Physique - Matière et Rayonnement, F-75005 \\ Paris, France. \\ ${ }^{2}$ Laboratory of Physics of Radiation and Laser-Matter Interactions, Faculty of Sciences. Moulay \\ Ismail University, BP11201, Zitoune Meknes, Morocco.
}

\begin{abstract}
We investigate the total cross sections of the electron transfer process in the protonhydrogen collisional system involving initial excited states at impact energies in the $2.5 \mathrm{eV} / \mathrm{u}-100 \mathrm{keV} / \mathrm{u}$ range. The calculations are based on two non-perturbative treatments, namely the semiclassical Atomic Orbitals Close-coupling (SC-AOCC) approach and the Classical Trajectory Monte Carlo (CTMC) method. The results are presented and discussed for the collisions $H^{+}+H(n=1,2,3)$ taking into account the degeneracy of each level in orbital momentum $\ell$. We find that the SC-AOCC results show empirical $n^{3}$ and $n^{4}$ scaling laws at low and high impact velocities, respectively.
\end{abstract}

Keywords: electron transfer, ion-atom collision, non-perturbative treatments. 


\section{Introduction}

The capture process in ion-atom collisions has attracted special interest over many decades [1], due to its utmost importance in various domains, from fusion to interstellar space research [2]. For example the corresponding cross sections are key input data for plasma fusion since they are required in collisional radiative modelling and diagnostics tools, as neutral beam spectroscopy [3], as stored e.g. in the Atomic Data and Analysis Structure (OPEN-ADAS) [4].

As a benchmark system, the capture in collisions between proton and ground-state hydrogen $\mathrm{H}(1 \mathrm{~s})$ has been extensively investigated both experimentally [5-13] and with quantal, classical and semiclassical approaches developed to understand the general behavior of electron transfer processes in ion-atom collisions [14-18].

On the other hand the electron capture from excited hydrogen $H(n=2)$ by various ions has been treated using, among others, two non-perturbative approaches: the Classical Trajectory Monte Carlo (CTMC) method [19] and the semiclassical Atomic Orbitals Close-coupling (SC-AOCC) approach [20]. In the very low velocity region where the resonant capture is extremely dominant, the electron transfer process was investigated for $H^{+}+H(n l)$ within a pure quantal treatment, in which the total wave function is expanded in the basis set of the eigenstates of the transient molecule formed during the collision [2123]. With increasing collision velocity, a pure quantal study becomes more computationally demanding and semiclassical treatments are justified and tractable [24]: in the latter, the nuclear motion is treated classically and a quantum approach is used to describe the electronic part. In particular, the well-know semiclassical non-perturbative SC-AOCC is a good approximation in the intermediate velocity range [25$27]$.

However, for collisional systems involving very high excited initial hydrogen states, the numerical implementation of the SC-AOCC approach requires extremely large basis sets and therefore very large computation time, even for proton projectile which corresponds to the simplest collision system in ion-atom collisions. Alternatively, for highly excited targets, the CTMC method presents several advantages : (i) fundamentally, through Bohr's equivalence principle when $n \gg 1$, (ii) its fairly simple numerical implementation and (iii) much lower computational costs compared to the SC-AOCC approaches. In addition, the CTMC method is a good approximation at intermediate velocities to investigate electron transfer and ionization processes induced by impact of bare ions [28]. Note that CTMC has been recently used even for collisions between ions and complex targets, e.g., $N a(3 s), N a^{*}(3 p)$, $\mathrm{Li}(2 s), A r$ and $H e$, where the active electron is subject to non-coulomb interactions and requires the use of model potentials $[29,30]$

Nowadays the impressive progress of highlyparallel computers allows the use of the SC-AOCC approach with very large basis sets, which were unusable before due to prohibiting CPU execution time. Therefore, our purpose in this paper is to present a study of the capture processes for proton-hydrogen collisions involving initial excited states, up to $\mathrm{H}(\mathrm{n} \ell)$, using non-perturbative SC-AOCC and, for comparison, CTMC approaches. Indeed the CTMC gives accurate results in the intermediate velocity range [31] and it is expected to be a good approximation for highly excited targets even in the low velocity range. We also present comparisons with experimental data, when available, and with the analytical fit proposed by Janev et al. [32], based on many theoretical and experimental data depending on the collision velocity range (see [32] and references within). In addition, we compare our results with those used in plasma physics, available in the ADAS database [4].

The paper is organised as follows. In Sec. 2, we briefly review the CTMC method and the SC-AOCC approach that are used to obtain the results presented in Sec. 3. This is followed by their comparisons and a discussion which ends up by the writing of two empirical scaling laws and a general fitting function obtained from the SC-AOCC results for the shell selected capture cross sections. Finally, conclusions are presented in Sec. 4.

Atomic units (a.u.) are used throughout the paper unless stated otherwise.

\section{Theoretical approaches}

The proton-hydrogen collision system is a three-body problem formed by the electron $e$ of mass $m_{e}$, targetproton $T$ of mass $M_{p}$ and the projectile-proton $P$ of mass $M_{p}$. The $\overrightarrow{r_{e}}, \overrightarrow{r_{T}}$ and $\overrightarrow{r_{P}}$ denote the position vectors for the electron, target proton and for the proton projectile, respectively, in the laboratory frame, see Fig. 1. In order to reduce the three-body problem to a two-body problem, it is convenient to use the Jacobi coordinates $\left(\vec{r}, \vec{R}, \vec{R}_{C M}\right)$ where $\vec{r}$ is the coordinate of the electron with respect to the proton target, $\vec{R}$ the relative coordinate of the projectile with respect to the electron-target-proton center of mass and $\vec{R}_{C M}$ the centre-of-mass coordinate. In the center-of-mass frame, the three-body system reduces to a two-body problem with the corresponding Hamiltonian

$$
H=\frac{p^{2}}{2 \mu}+\frac{P^{2}}{2 M}+V_{T e}(r)+V_{P e}(x)+V_{P T}(s),
$$

where $\vec{p}$ and $\vec{P}$ are the momenta related to $\vec{r}$ and $\vec{R}$, respectively, and the reduced masses $\mu$ and $M$ are $\mu=$ $m_{e} M_{p} /\left(m_{e}+M_{p}\right)$ and $M=M_{p}\left(M_{p}+m_{e}\right) /\left(m_{e}+2 M_{p}\right)$. In Eq. $1, V_{T e}, V_{P e}$ and $V_{P T}$ are respectively, the electron-target, the electron-projectile and the protonproton Coulomb potentials with the corresponding 
separation distances $r, x$ and $s$ defined in Fig. 1 . In the following, we review the applied theoretical approaches used to study the capture processes for proton-hydrogen collisions.



Figure 1. (color online) Schematic illustration of the relation between the position vectors $\vec{r}_{e}, \vec{r}_{T}$ and $\vec{r}_{P}$ in the laboratory frame and the Jacobi coordinates $\vec{r}$ and $\vec{R}$, where the $e, T$ and $P$ denote the electron, the proton target and the proton projectile, respectively.

\subsection{Classical Trajectory Monte Carlo}

In order to calculate the cross sections for electron transfer processes classically in $H^{+}+H(n l)$ collisions, we use the Classical Trajectory Monte Carlo model proposed by Abrines and Percival [14]. The dynamics of the collision system is determined by the classical Hamilton equations

$$
\dot{r}_{i}=\frac{p_{i}}{\mu} \quad, \quad \dot{p}_{i}=-\frac{\partial H}{\partial r_{i}}, \quad i=1,2,3
$$

and

$$
\dot{R}_{i}=\frac{P_{i}}{M} \quad, \quad \dot{P}_{i}=-\frac{\partial H}{\partial R_{i}}, \quad i=1,2,3
$$

where the subscript $i$ denotes the three Cartesian coordinates. The system of equations (2) is solved numerically by the means of a Runge-Kutta method with an adaptive step size, starting from a given configuration of the collision partners which defines the initial conditions $\left(\vec{r}^{0}, \vec{p}^{0}\right)$ and $\left(\vec{R}^{0}, \vec{P}^{0}\right)$. In order to represent classically an initial state $H(n \ell)$ of principal quantum number $n$ and angular momentum $\ell$, we have chosen to use the Becker and MacKellar approach [33] which generalises the procedure of Abrines and Percival by subdividing the initial microcanonical ensemble (for a complete $n$ shell) into sub-ensembles corresponding to the quantal state $H(n \ell)$. Each initial electronic trajectory is then defined by five random parameters: the ellipse eccentricity $\varepsilon$, where $\varepsilon^{2}$ is sampled uniformly in the interval $\left[1-\frac{(\ell+1)^{2}}{n^{2}}, 1-\frac{\ell^{2}}{n^{2}}\right]$, the mean anomaly $\alpha$, where $0 \leq \alpha \leq 2 \pi$, and the three Euler angles $(\psi, \theta, \phi)$, which determine the random orientation of the ellipse in space. The eccentric angle $\xi$ is defined from the parameters $\alpha$ and $\varepsilon$ by solve the Kepler's equation

$$
\alpha=\xi-\varepsilon \sin (\xi) .
$$

Then the vectors $\overrightarrow{r^{0}}$ and $\overrightarrow{p^{0}}$ can be obtained in terms of the energy $E_{n}=-1 / n^{2}, \xi, \varepsilon$, and the three Euler angles.

In order to initialize the incident projectile, we consider the collision frame illustrated in Fig. 2 for an azimuthal angle $\varphi_{b}=0$, i.e., the collision plane is the (XOZ) plane. The projectile is specified by its momentum $\vec{P}^{0}=M \overrightarrow{\mathrm{v}}$ where the relative velocity $\overrightarrow{\mathrm{v}}$ is chosen along $\mathrm{Z}$ and its initial distance $R^{0}$ from the electron target-proton center-of-mass is set sufficiently large to be able to consider that the two collision partners are isolated. The uniform beam of proton projectile is constructed by choosing randomly the square of the impact parameter $\vec{b}$ in the interval $\left[0, b_{\max }^{2}\right]$ where $b_{\max }$ is the value of the impact parameter beyond which the studied processes do not take place. Its value varies depending on the process, the collisional target and the collision velocity.

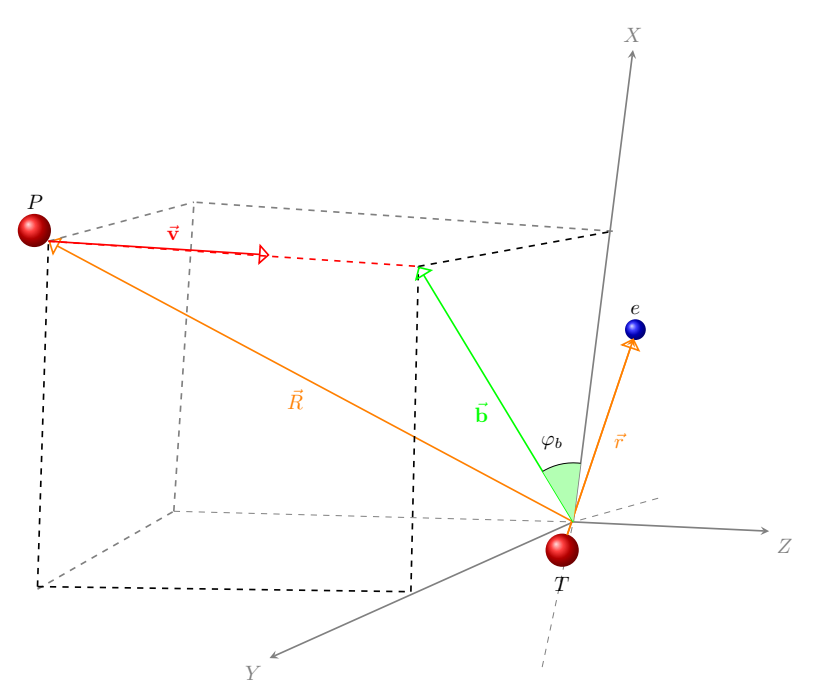

Figure 2. (color online) The collision frame and geometry: the collision plane is defined by the vectors $\vec{R}, \overrightarrow{\mathrm{v}}$ and $\vec{b}$. In our case, the chosen collision plane is the $(X O Z)$ one, i.e., the relative velocity $\overrightarrow{\mathrm{v}}$ is chosen along the $Z$ axis and the impact parameter $\vec{b}$ along $X$

For a given set of initial conditions $\left(\vec{r}^{0}, \vec{p}^{0}\right)$ and $\left(\overrightarrow{R^{0}}, \vec{P}^{0}\right)$, the set of the twelve coupled equations, resulting from (2), is solved numerically. This procedure is repeated for a large number of trajectories, $N_{\text {tot }}$, in order to reach the reasonable statistical convergence of the cross sections. 
During the interaction between the projectile and the hydrogen target, the electron moves under the influence of the potentials, $V_{T e}$ and $V_{P e}$ so that, at the end of the collision, three different processes can be distinguished. The first kind of process corresponds to the electron which remains bound to the target, i.e., an elastic or excitation process has occurred. The second process is when the electron is captured by the proton projectile (electron transfer). In this case, the electron passes from a bound state on the hydrogen target to a bound state on the proton projectile so that its energy relative to the projectile ion becomes negative while its energy with respect to the target gets positive. Thirdly, if none of these processes happen, ionization takes place.

For a given collision velocity the total cross section (TCS) of a process $\tau$ (capture, ionization or excitation) in the $H^{+}+H(n \ell)$ collision is given by

$$
\sigma_{\tau}^{n \ell}(\mathrm{v})=\frac{N_{\tau}^{n \ell}}{N_{t o t}} \pi b_{\max }^{2}
$$

and for a reaction $H^{+}+H(n)$

$$
\sigma_{\tau}^{n}(\mathrm{v})=\sum_{\ell=0}^{n-1} \frac{(2 \ell+1) \sigma_{\tau}^{n \ell}(\mathrm{v})}{n^{2}}
$$

where $N_{\tau}^{n \ell}$ is the number of events of the process $\tau$. We have run many tests to determine the appropriate $b_{\max }$ for each $H^{+}+H(n \ell)$ collision and for each considered velocity $v$. For example, the capture process from excited hydrogen target may be likely for large values of $b_{\max }$ in low velocity range: $b_{\max }=20$ a.u. for $H(2 \ell)$ targets, and $b_{\max }=48$ a.u. for $H(3 s), H(3 p)$ and $H(3 d)$ targets.

\subsection{The semiclassical Atomic Orbitals Close-Coupling}

The scattering problem is treated by the semi-classical atomic orbital close-coupling approach within the impact parameter approximation [24]. The relative target-projectile motion is described by straightline, constant velocity, trajectories where the position vector $\vec{R}$ reads,

$$
\vec{R}=\vec{b}+\overrightarrow{\mathrm{v}} t .
$$

while the electronic motion is described quantally through the time-dependent Schrödinger equation

$$
\left[H-i \frac{\partial}{\partial t}\right] \psi(\vec{r}, t)=0
$$

In our approach, see [34,35], the equation (7) is solved as follows: the electronic wave function $\psi$ is expanded into target and projectile-centered traveling atomic orbitals $\xi_{k}^{T}$ and $\xi_{k}^{P}$, where $k$ denotes the quantum numbers $n, \ell$ and $m$ [25-27]. The electronic wave function $\psi$ is thus written as

$$
\psi(\vec{r}, t)=\sum_{k=1}^{N_{T}} a_{k}^{T}(t) \xi_{k}^{T}(\vec{r}, t)+\sum_{k=1}^{N_{P}} a_{k}^{P}(t) F(\vec{r}, t) \xi_{k}^{P}(\vec{x}, t),
$$

where

$$
F(\vec{r}, t)=e^{i \vec{v} \cdot \vec{r}-i \frac{1}{2} \mathrm{v}^{2} t}
$$

denotes the Electron Translation Factor (ETF) [24] which ensures the Galilean invariance of the results. The time-dependent complex amplitudes $a_{k}^{T}$ and $a_{k}^{P}$ related to the states $\xi_{k}^{T}$ and $\xi_{k}^{P}$, respectively, have to be obtained numerically and become probability amplitudes in the asymptotic regions $(t \rightarrow \infty)$ where all states in the expansion (8) are orthogonal.

The calculations are performed in the collision frame as considered in the CTMC method, i.e., the impact parameter $\vec{b}$ is parallel to $X$ and the velocity $\overrightarrow{\mathrm{v}}$ is parallel to the quantization axis $Z$.

The first step is to obtain numerically the eigenstates (and pseudo-states) $\xi_{k}^{T, P}$ and their associated eigenvalues (positive energies) by diagonalizing the Hamiltonians $h_{T}=H-V_{P e}-V_{P T}$ and $h_{P}=H-V_{T e}-V_{P T}$, respectively. In our calculations, we used a basis set consisting of 160 states of the target and projectile hydrogen expressed in terms of Gaussian type orbitals $s$, $p, d, f$ and $g$, on each center. Energetically, this basis set describes well the bound states up to the level $n=7$ with negligible differences with the exact energies. The complex amplitudes $a_{k}^{T}$ and $a_{k}^{P}$ are obtained asymptotically by solving numerically the equation (7) along a certain nuclear trajectory $(b, v)$. The transition probability from the initial state $|n \ell m\rangle$ to another state $\left|n^{\prime} \ell^{\prime} m^{\prime}\right\rangle$ leading to the electronic process $\tau$ under consideration is defined as follows

$$
P_{\tau}^{n \ell m \rightarrow n^{\prime} \ell^{\prime} m^{\prime}}(b, \mathrm{v})=\left|a_{n^{\prime} \ell^{\prime} m^{\prime}}^{K}(t \rightarrow+\infty)\right|^{2},
$$

where $a_{n^{\prime} \ell^{\prime} m^{\prime}}^{K}$ denotes the complex amplitudes $a_{n^{\prime} \ell^{\prime} m^{\prime}}^{T}$ or $a_{n^{\prime} \ell^{\prime} m^{\prime}}^{P}$. The partial cross section of an electronic process $\tau$ for a given velocity corresponding to the transition $|n \ell m\rangle \rightarrow\left|n^{\prime} \ell^{\prime} m^{\prime}\right\rangle$ reads

$$
\sigma_{\tau}^{n \ell m \rightarrow n^{\prime} \ell^{\prime} m^{\prime}}(\mathrm{v})=2 \pi \int_{0}^{b_{\max }} P_{\tau}^{n \ell m \rightarrow n^{\prime} \ell^{\prime} m^{\prime}}(b, \mathrm{v}) b d b .
$$

Then, the total cross section of a process $\tau$ is given by

$$
\sigma_{\tau}^{n \ell m}(\mathrm{v})=\sum_{n^{\prime} \ell^{\prime} m^{\prime}} \sigma_{\tau}^{n \ell m \rightarrow n^{\prime} \ell^{\prime} m^{\prime}}(\mathrm{v})
$$

Note that in the case of the capture processes, the values of $b_{\max }$ established in the SC-AOCC approach are larger than those found in the CTMC approach for a given collision velocity. This is mainly due the quantum description of the electronic motion 
which does not possess the cut-off in the probability density inherent to the classical description of bound trajectories around an attraction center.

Convergence of the cross sections with respect to the basis set was carefully checked by using a larger basis set composed of 191 states on each center: it was found that the cross sections evaluated with the large basis do not differ by more than $3 \%$ from those obtained with the first basis for all impact energies considered in this work, for the initial targets $H(n=1,2)$. However for target $\mathrm{H}(\mathrm{n}=3)$, the difference between both basis sets at low and high velocities does not exceed $4 \%$ while at intermediate velocities the difference can reach about $10 \%$.

\section{Results and discussion}

\subsection{Total cross sections of capture in $H^{+}+H(1 s)$} collisions
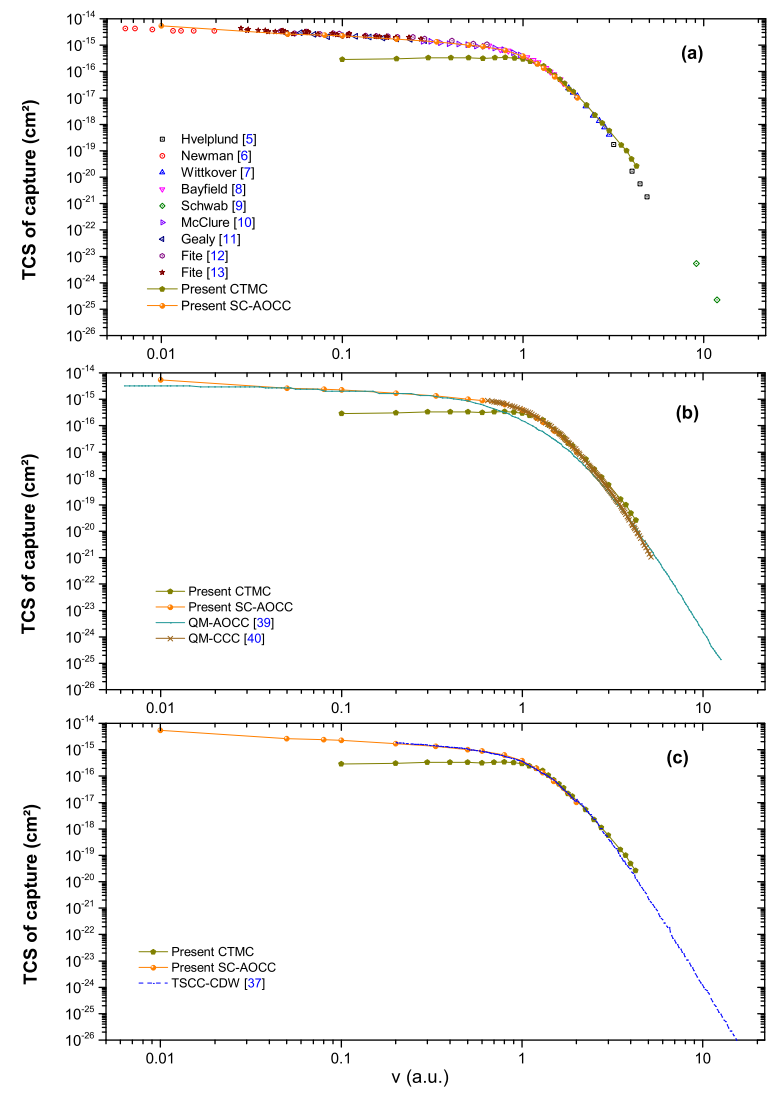

Figure 3. (color online) Total cross section of capture from $\mathrm{H}(1 \mathrm{~s})$ by protons as function of the relative velocity $\mathrm{v}$.

Fig. 3a shows the comparison between the total cross sections of the capture process obtained with our CTMC and SC-AOCC approaches and the available experimental data. In the intermediate velocity range $(\mathrm{v} \approx 1$ a.u. $)$, the present $\mathrm{CTMC}$ results are in agreement with those of the SC-AOCC and with the experimental data reported in the references [5-13]. In the low velocity range $(\mathrm{v}<1$ a.u. $)$, the CTMC cross sections show a serious discrepancy, showing a nearly constant value, thus underestimating the experimental and semiclassical data. For initial ground state target, a quantum or semiclassical treatment is therefore needed in this latter region where molecular mechanisms play a dominant role, not described by classical mechanics.

The present SC-AOCC results are in excellent agreement with those found experimentally in the all velocity region considered, $0.01<\mathrm{v}<2$ a.u. In the low velocity range, the resonant capture, for which the energy transferred to the electron is almost zero, is dominant and well taken into account by the SC-AOCC approach. The observed very good agreement with the experimental data suggests also that even for the lowest velocities considered, the nuclear relative motion can be treated classically, see Eq. (6). This was also demonstrated by Hunter et al. [36] by comparing their purely quantum results (Perturbed Stationary States in Partial Wave treatment) with the semiclassical results of Bates et al. [37].

In Fig. 3b our results are compared with two recent close-coupling calculations $[38,39]$. In [38] the authors have used a fully quantum-mechanical approach based on the atomic orbital close coupling method (QM-AOCC). Their TCS are in agreement with our present SC-AOCC results and with the experiments except in the intermediate velocity range $(0.2<\mathrm{v}<2$ a.u $)$. Results in [39], obtained with the quantum-mechanical convergent close-coupling method (QM-CCC) using Laguerre basis functions, are in excellent agreement with our semiclassical results in the intermediate velocity range.

It should be mentioned that the SC-AOCC approaches as well as, intrinsically, CMTC method do not formally satisfy the boundary conditions, as defined in the most refined continuum disturbed waves theories, see for example [40-42]. To evaluate the effects of the boundary conditions, we compare in Fig. 3c our results with those of da Silva et al. [43]. These authors have calculated the cross section of the resonant capture $1 \mathrm{~s}-1 \mathrm{~s}$ using two-state close-coupling approximation with the continuum distorted wave basis set (TSCC-CDW) proposed by Cheshire [40], which satisfies the boundary conditions. Fig. 3c shows our cross sections for total capture with those reported in [43] and deduced from their resonant capture cross sections by using the scaling rule $\sigma_{\text {capture }}^{1 s \rightarrow \text { all states } \approx}$ $1.202 \sigma_{\text {capture }}^{1 s \rightarrow 1 s}$ proposed by Oppenheimer [44]. These TCS are in excellent agreement with the present SC-AOCC results in the overlapping velocity range, 
showing negligible effects of the boundary conditions in this energy range.

\subsection{Total cross sections of capture in $H^{+}+H(n=2)$ collisions}

The electron capture processes from excited states have been much less investigated. To compare our results, we use the available results for capture from $H(2 s)$ and $H(2 p)$ targets from the ADAS database which uses two series of theoretical data: at high velocities, the results of Eichler [45], based on the perturbative eikonal theory, and, for velocities in the low and intermediate ranges, the SC-AOCC results of Reinhold and Falcon [46].

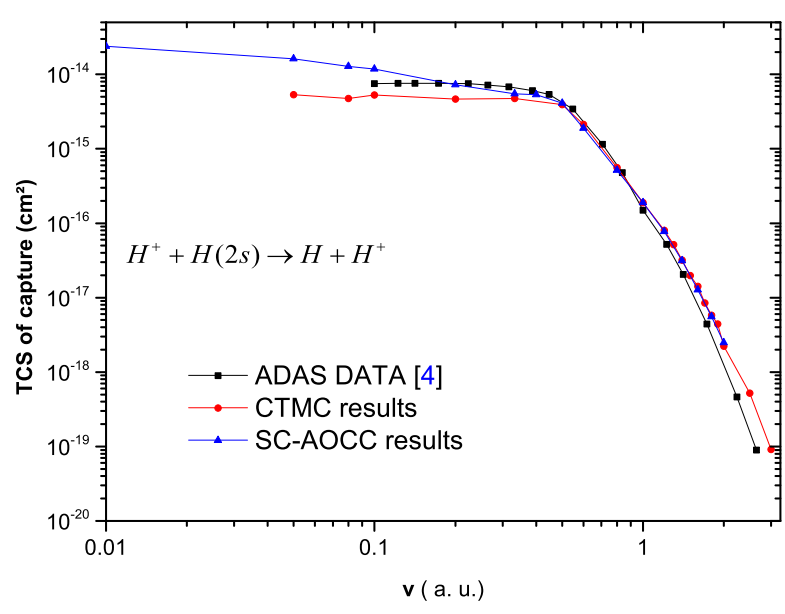

Figure 4. (color online) TCS of capture from $H(2 s)$ by protons as function of the relative velocity $\mathrm{v}$.

In Figs. 4 and 5, we present the capture TCS for the excited targets $H(2 s)$ and $H(2 p)$, respectively. In the intermediate velocity range, down to $\mathrm{v} \approx 0.5$ a.u., the present SC-AOCC results are in a good agreement with those obtained by the CTMC approach, but there is a significant disagreement with those stored in ADAS [4], especially for $H(2 p)$ initial state, certainly related to the limited basis set used in [46]. At low velocities ( $\mathrm{v}<0.5$ a.u. $)$, there is a pronounced discrepancy between the SC-AOCC results and those of CTMC corresponding to $H(2 s)$ and $H(2 p)$ targets. This disagreement between the quantum and classical results becomes larger for very low velocities. Note however that the discrepancy is less pronounced that the one shown in Fig. 3 for $\mathrm{H}(1 \mathrm{~s})$ initial state. In the high velocity region ( $\mathrm{v}>1$ a.u.), in the case of $H(2 s)$ excited target, the TCS of capture stored in ADAS database decay faster than those obtained with the CTMC and SC-AOCC methods whereas for the $H(2 p)$ target the ADAS data are closed to those obtained with



Figure 5. (color online) TCS of capture from $H(2 p)$ by protons as function of the relative velocity $\mathrm{v}$.

the present two approaches.

In order to compare the semiclassical and the CTMC results with the analytical fit of Janev [32], we calculate the average capture cross sections (see Eq. 5) from all possible quantum states of the energy level $n=$ 2. The results are shown in Fig. 6. We note that in the regime of low and intermediate velocities, our SC-AOCC results are in agreement with those recommended by the analytical fit while the CTMC results and the ADAS data underestimate the TCS of capture. At high velocities the analytical fit decreases faster than those calculated by CTMC and SC-AOCC approaches.

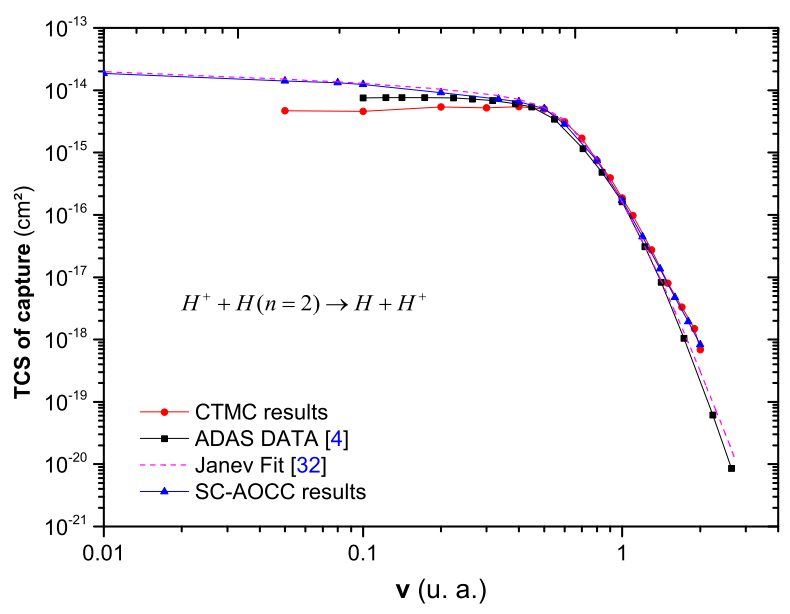

Figure 6. (color online) TCS of capture from $H^{*}(n=2)$ by protons as function of relative velocity v. 
3.3. Total cross sections of capture in $\mathrm{H}^{+}+H(n=3)$ collisions

To our knowledge, there is no non-perturbative calculations published for the capture TCS from the excited states $H(3 s), H(3 p)$ and $H(3 d)$. In absence of theoretical and experimental data, we compare in Figs. 7,8 , and 9 the present SC-AOCC results only with the CTMC ones.

At low velocities, ie. $\mathrm{v}<\mathrm{v}_{e}^{n=3} \approx 0.333$ a.u. which corresponds to the matching velocity regime where the projectile velocity is close to the average electronic velocity in the initial state, we note that the present CTMC results for the capture TCS from $H(3 \ell)$ are again lower than the semiclassical results. In contrast, a good agreement between both approaches is found in the intermediate velocity range $\left(\mathrm{v} \approx \mathrm{v}_{e}^{n=3}\right)$. At high velocities $\left(\mathrm{v}>\mathrm{v}_{e}^{n=3}\right)$, for capture from the excited $H(3 s)$ and $H(3 p)$ sub-shells, a good agreement is found between the SC-AOCC and the CTMC results. For the capture from $H(3 d)$, the CTMC results decrease faster than those obtained by the SC-AOCC approach.

In order to compare the results from these three sub-shells, we report in Fig. 10 the capture cross sections for $H(3 \ell)$ initial states obtained with the SCAOCC approach. First, in the low velocity range, the three series of TCS are of the same order of magnitude for all quantum states. At intermediate velocities, the capture from the state $H(3 d)$ is more likely than the capture from the excited state $H(3 p)$, which is larger than the capture from the state $H(3 s)$. This latter behaviour is opposite and more pronounced in the case of high velocities. Therefore we can write,

$$
\left\{\begin{array}{lc}
\sigma_{c}^{3 d}(\mathrm{v}) \approx \sigma_{c}^{3 p}(\mathrm{v}) \approx \sigma_{c}^{3 s}(\mathrm{v}) & (\mathrm{v} \ll 0.333 \text { a.u. }) \\
\sigma_{c}^{3 d}(\mathrm{v})>\sigma_{c}^{3 p}(\mathrm{v})>\sigma_{c}^{3 s}(\mathrm{v}) & (\mathrm{v} \approx 0.333 \text { a.u. }) \\
\sigma_{c}^{3 d}(\mathrm{v})<\sigma_{c}^{3 p}(\mathrm{v})<\sigma_{c}^{3 s}(\mathrm{v}) & (\mathrm{v} \gg 0.333 \text { a.u. })
\end{array}\right.
$$

where $\sigma_{c}^{3 s}, \sigma_{c}^{3 p}$ and $\sigma_{c}^{3 d}$ are the capture TCS from the excited states $H(3 s), H(3 p)$ and $H(3 d)$, respectively.

In Fig. 11, we present the classical and semiclassical results for the capture averaged TCS from the $H(n=$ 3) shell, together with the fit derived in Janev et al. [32] and the ADAS data, ie. data obtained by a perturbative eikonal theory [45]. Our semiclassical results agree well at intermediate and low velocities with the fit [32] and with those stored in ADAS [4]. As noted before, the CTMC approach underestimates the TCS at low velocities, but performs well at higher velocities. On the other hand, in the high velocity range, the capture TCS from the fit decrease faster than the CTMC and semiclassical results.

It is important to note that at low velocities, the discrepancy between classical and semiclassical TCS of capture becomes less and less important when considering higher and higher excited initial

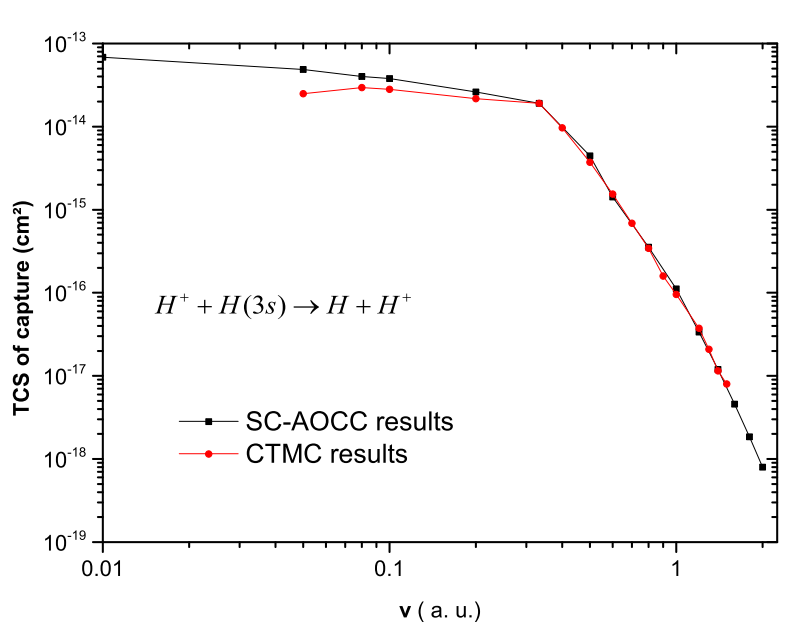

Figure 7. (color online) TCS of capture from the excited state $H(3 s)$ by protons as function of relative velocity $\mathrm{v}$.

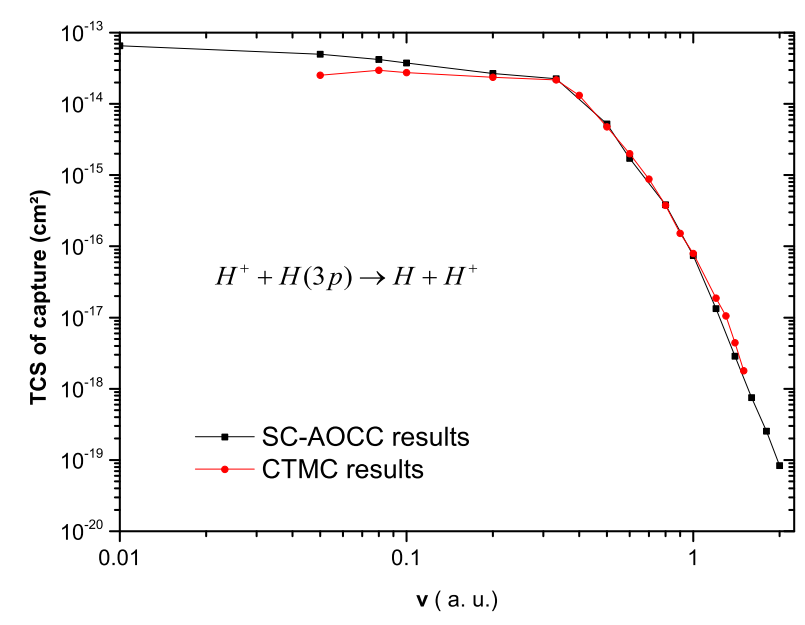

Figure 8. (color online ) TCS of capture from the excited state $H(3 p)$ by protons as function of relative velocity $\mathrm{v}$.

targets. It is thus expected that this disagreement becomes negligible for capture from highly excited states. In these cases, the CTMC can therefore be used even at low velocities. On the other hand the semiclassical calculations of capture cross section from higher excited initial states $H(n>3)$ need larger basis sets that the one we employ here. In particular, it should include higher angular momenta $(\ell>4)$ so that the SC-AOCC approach cannot currently be used in a reasonable CPU time. These are the reasons why we only used the CTMC approach to investigate capture from high excited states, $H(n>3)$. In Fig. 12 , we present the corresponding cross sections for capture from the $H(n=1, \ldots, 7)$ targets. We can 



Figure 9. (color online) TCS of capture from the excited state $H(3 d)$ by protons as function of relative velocity $\mathrm{v}$.

Figure 11. (color online) TCS of capture from $H(n=3)$ by protons as function of relative velocity $\mathrm{v}$.


Figure 10. (color online) TCS of capture from the excited states $H(3 s), H(3 p)$ and $H(3 d)$ by protons as function of relative velocity $\mathrm{v}$.

obviously remark that the TCS of capture from any state $H(n \leq 7)$ has a similar behaviour which will be analysed in the next section.

\section{4. $n^{3}$ and $n^{4}$ scaling laws for total cross sections of} capture from $H(n)$ by protons

In Fig. 13, we present the semiclassical TCS scaled in $n^{3}$ as a function of the scaled velocity $\tilde{\mathrm{v}}=\mathrm{v} / \mathrm{v}_{e}^{n}$, where $\mathrm{v}_{e}^{n}=1 / n$ is the velocity of the electron target on the Bohr orbit corresponding to the energy level $n$. The present semiclassical non-perturbative treatment shows that the TCS of capture from the excited states $H(n=2,3)$ follow fairly well an empirical scaling in

Figure 12. (color online) TCS of capture from $H(n=1-7)$ by protons as function of relative velocity $\mathrm{v}$ obtained by the microcanonical CTMC.

$n^{3}$ at low velocities $(\tilde{\mathrm{v}}<1)$. This behaviour was first predicted by Bates and Reid in an earlier work [22] using a two-state molecular quantal treatment. The authors have performed the calculations for resonant capture from initial states up to the $H(n=5)$ shells; they report that the cross sections of the resonant electron transfer can be written as

$$
\sigma_{c}^{n \rightarrow n}(\mathrm{v})=n^{3} \mathcal{A}(\mathrm{v}) \quad(n \geq 2)
$$

where $\mathcal{A}$ is a function of the velocity v. On the other hand, as shown in Fig. 14, the TCS of capture follow a scaling law in $n^{4}$ in the region of intermediate and high velocities, ie. $(\tilde{\mathrm{v}} \geq 1)$. The capture TCS 


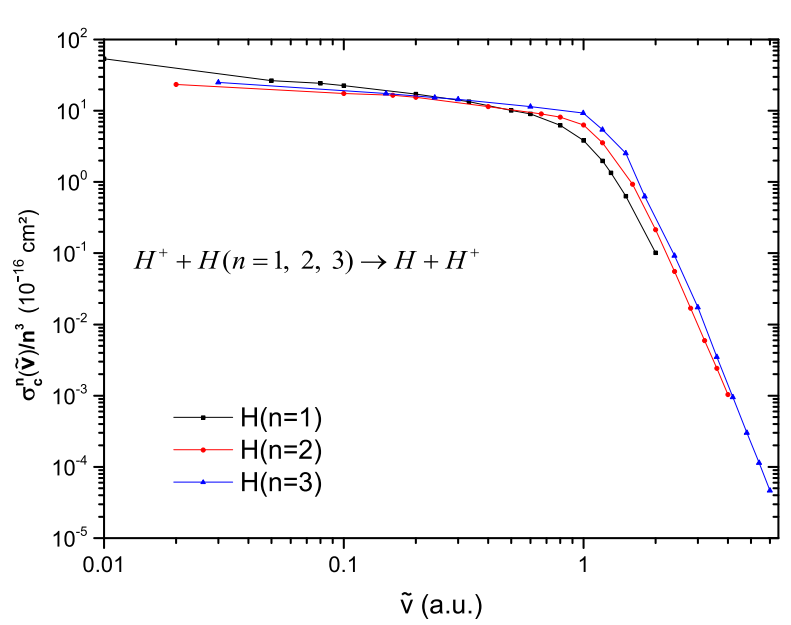

used to predict the TCS of electron capture in a wide range of impact velocities including low, intermediate and high velocity regimes. The proposed analytical TCS reads,

$\sigma_{c}^{n}(\mathrm{v})=n^{3}\left(1-g^{n}(\mathrm{v})\right) \mathcal{A}^{n}(\mathrm{v})+n^{4} g^{n}(\mathrm{v}) \mathcal{B}^{n}(\mathrm{v}) \quad\left[10^{-16} \mathrm{~cm}^{2}\right]$

where $g^{n}(\mathrm{v})=1 /\left(1+e^{-\alpha(\mathrm{v}-1 / n)}\right)$ and $\mathrm{v}$ is in atomic unit. In order to find the appropriate functions $\mathcal{A}^{n}$ and $\mathcal{B}^{n}$, we employed the analytical functions used in [32]:

$$
\begin{aligned}
& \mathcal{A}^{n}(\mathrm{v})=4.8 \log \left(2 \frac{n^{2}}{\mathrm{v}^{2}}+10^{n-2}\right), \quad \mathrm{v}<\mathrm{v}_{e} \\
& \mathcal{B}^{n}(\mathrm{v})=\frac{A}{1+B(n \mathrm{v})^{2}+C(n \mathrm{v})^{7.2}+D(n \mathrm{v})^{11}}, \quad \mathrm{v} \geq \mathrm{v}_{e}
\end{aligned}
$$

where the constants $\mathrm{A}, \mathrm{B}, \mathrm{C}$ and $\mathrm{D}$ are all positive, with $A=7, B=0.75, C=0.4$ and $D=9.7710^{-4}$. As a final note we would like to mention that we found that the TCS from [32] decrease in the high velocity range faster than our semiclassical results (see Figs. 6 to 11), failure which is corrected in the present fit. However in this latter region, the analytical fit of Janev et al. [32] is only based on the theoretical data of Eichler [45] which were obtained by the eikonal perturbative treatment. Alternatively, the proposed analytical fitting (Eq. 16) shows a good agreement with the exact SC-AOCC results for all velocities, as shown in the Figs. 15a and 15b, which can validate this latter fit: the difference of the TCS evaluated by our fit with respect to the calculated SC-AOCC results for the initial states $H(n=2)$ and $H(n=3)$ does not exceed $15 \%$ in the high and intermediate velocities and about $10 \%$ at low velocities.

As a final task to check the validity of the proposed fit, we compare in Figs. 15c and 15d the TCS obtained with our fit and those calculated with the CTMC method, for the excited states $H(n=4)$ and $H(n=7)$. As expected, in the intermediate and high velocities, the fit is in a good agreement with the classical results for these highly excited states. Compared to the excited states $H(n=2-4)$, the TCS of capture from the excited state $H(n=7)$ by protons are in excellent agreement with those established by CTMC at low velocities where the discrepancy between the approaches manifests only towards very low velocities, below to 0.04 a.u. $(40 \mathrm{eV} / \mathrm{u})$.

\section{Conclusions}

In this paper we have presented total cross sections corresponding to the capture processes from excited atomic hydrogen targets $H(n=2,3)$ by impact of protons, using two non-perturbative approaches (CTMC and SC-AOCC) at impact velocities in the 

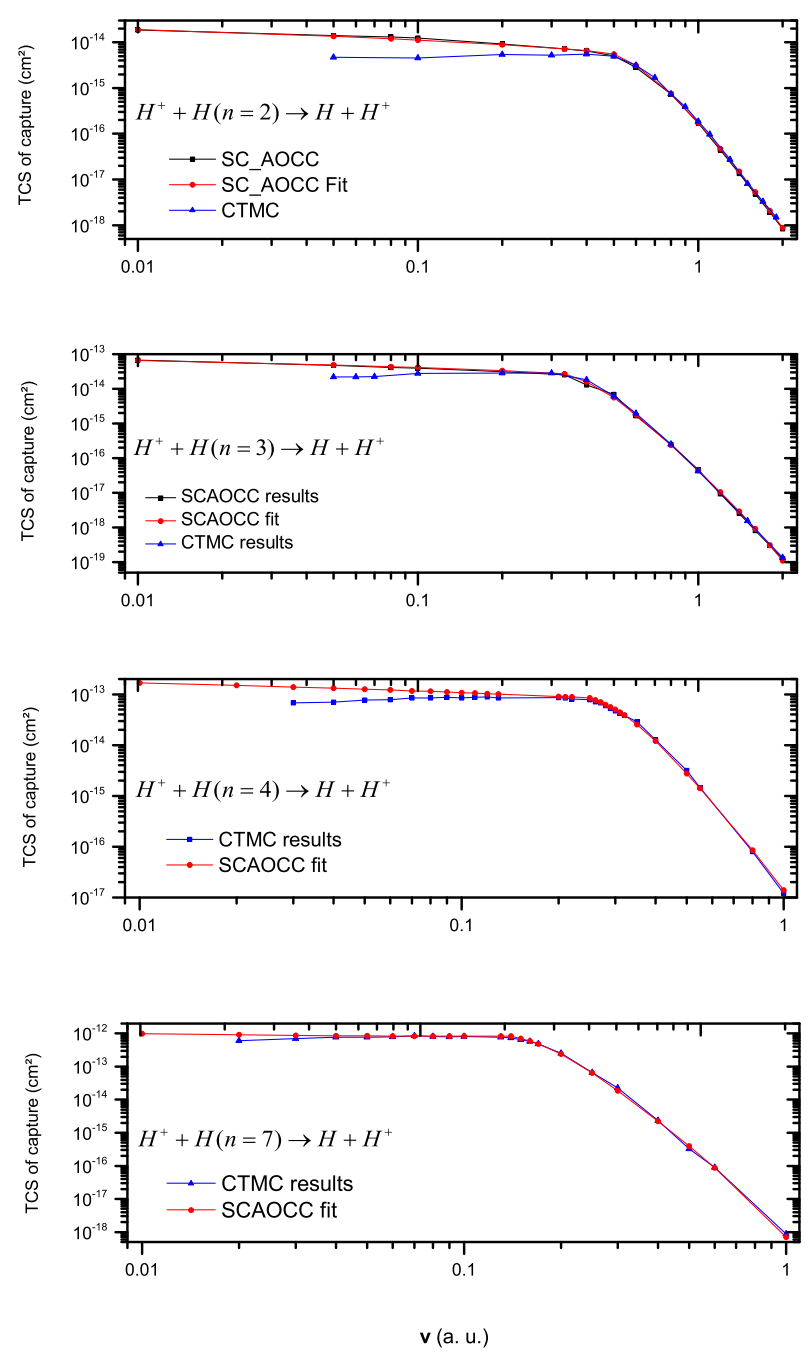

Figure 15. (color online) The comparison between the proposed fit and CTMC results.

(0.01 - 2)a.u. range. The non-perturbative SC-AOCC provides accurate results in the low, intermediate and high velocity regions. For capture from higher excited targets $H(n>3)$ the SC-AOCC cannot currently be employed due to prohibiting CPU execution time. Alternatively, we searched for an analytical fit according to the SC-AOCC results in order to overcome this numerical limitation. The proposed analytical fit for capture TCS is relevant in the whole range of impact energies considered in this work and gives approximatively the prediction of SC-AOCC for any $H(n)$ exited target.

At intermediate and high velocities for the excited targets $H(n=2,3)$ we show an excellent agreement between the present non-perturbative classical and semiclassical treatments. Furthermore, at low velocities, the discrepancy between classical and semiclassical capture TCS becomes less important when considering higher and higher excited targets. Therefore, in the case of capture from excited states $H(n \gg 1)$, the CTMC can be considered as a solid approach, not only for the intermediate and high velocities [47] but also for low velocities, following the corresponding shift of the matching velocity $\mathrm{v}_{e}^{n}$. Finally, we should mention that the cross sections for initial $H(n=2,3)$ target stored in ADAS should be updated especially for the low and high velocity ranges.

\section{Acknowledgments}

AT was supported by a grant of Institut de Recherche pour le Développement IRD Bondy-France. This work was partly supported by LABEXs Plas@par and MiChem under Grant No. ANR-11-IDEX-0004-02.

\section{References}

[1] Ullrich J, Moshammer R, Dorn A, Drner R, Schmidt L P H and Schmidt-Bcking H 2003 Reports on Progress in Physics 661463

[2] Isler R, Crume E, Horton L, Murakami M, Baylor L, Bell G, Bigelow T, England A, Glowienka J, Jernigan T, Langley R, Mioduszewski P, Rasmussen D, Simpkins J, Wilgen J and Wing W 1991 Nuclear Fusion 31245

[3] Delabie E and et al 2010 Plasma Physics and Controlled Fusion 52125008

[4] Atomic data and analysis structure http://open.adas.ac. uk/

[5] Hvelplund P and Andersen A 1982 Physica Scripta 26375

[6] Newman J, Cogan J, Ziegler D, Nitz D, Rundel R, Smith K and Stebbings R 1982 Physical Review A 252976

[7] Wittkower A, Ryding G and Gilbody H 1966 Proceedings of the Physical Society $\mathbf{8 9} 541$

[8] Bayfield J E 1969 Phys. Rev. 185(1) 105-112

[9] Schwab W, Baptista G, Justiniano E, Schuch R, Vogt H and Weber E 1987 Journal of Physics B: Atomic and Molecular Physics 202825

[10] McClure G 1966 Physical Review 14847

[11] Gealy M and Van Zyl B 1987 Physical Review A 363091

[12] Fite W L, Stebbings R F, Hummer D G and Brackmann R T 1960 Physical Review 119663

[13] Fite W L, Smith A C and Stebbings R F 1962 Proc. R. Soc. Lond. A 268 527-536

[14] Abrines R and Percival I C 1966 Proceedings of the Physical Society $\mathbf{8 8} 861$

[15] Olson R and Salop A 1977 Physical Review A 16 531-541

[16] Bransden B H and McDowell M R C 1992 Charge exchange and the theory of ion-atom collisions (Clarendon Press)

[17] Belkić D 2003 Principles of quantum scattering theory (CRC Press)

[18] Belkić D 2008 Quantum theory of high-energy ion-atom collisions (CRC Press)

[19] Jorge A, Errea L F, Illescas C and Méndez L 2014 The European Physical Journal D 68 1-11

[20] Igenbergs K, Schweinzer J, Veiter A, Perneczky L, Frhwirth E, Wallerberger M, Olson R E and Aumayr F 2012 Journal of Physics B: Atomic, Molecular and Optical Physics 45065203

[21] Boyd T and Dalgarno A 1958 Proceedings of the Physical Society $\mathbf{7 2} 694$

[22] Bates D and Reid R 1969 Journal of Physics B: Atomic and Molecular Physics 2851 
[23] Malaviya V 1970 Journal of Physics B: Atomic and Molecular Physics 31492

[24] Bates D and McCarroll R 1958 Proceedings of the Royal Society of London A: Mathematical, Physical and Engineering Sciences vol 245 (The Royal Society) pp $175-183$

[25] Gallaher D and Wilets L 1968 Physical Review 169139

[26] Shakeshaft R 1976 Physical Review A 141626

[27] Fritsch W and Lin C D 1991 Physics Reports 202 1-97

[28] Abrines R and Percival I C 1966 Proceedings of the Physical Society $\mathbf{8 8} 873$

[29] Otranto S, Hoekstra R and Olson R E 2014 Physical Review A 89022705

[30] Focke P, Olson R, Cariatore N, Alessi M and Otranto S 2017 Physical Review A 95052707

[31] Olson R, Wang J and Ullrich J 1993 Physics of electronic and atomic collisions:XVIII International Conference Book Series: AIP Conference Proceedings 295 520-533

[32] Janev R K, Reiter D and Samm U 2003 Collision processes in low-temperature hydrogen plasmas (Forschungszentrum Jülich, Zentralbibliothek

[33] Becker R L and MacKellar A D 1984 Journal of Physics B: Atomic and Molecular Physics 173923

[34] Ibaaz A, Hernandez R, Dubois A and Sisourat N 2016 Journal of Physics B: Atomic, Molecular and Optical Physics 49085202

[35] Gao J, Wu Y, Sisourat N, Wang J and Dubois A 2017
Physical Review A 96052703

[36] Hunter G and Kuriyan M 1977 Proceedings of the Royal Society of London A: Mathematical, Physical and Engineering Sciences vol 353 (The Royal Society) pp 575-588

[37] Bates D and Boyd A H 1962 Proceedings of the Physical Society 801301

[38] Kadyrov A, Bray I and Stelbovics A 2006 Physical Review A 73012710

[39] Abdurakhmanov I, Kadyrov A, Avazbaev S and Bray I 2016 Journal of Physics B: Atomic, Molecular and Optical Physics 49115203

[40] Cheshire I 1964 Proceedings of the Physical Society 8489

[41] Belkić D, Gayet R and Salin A 1979 Physics Reports 56 279-369

[42] Belkić D, Mančev I and Hanssen J 2008 Reviews of Modern Physics $\mathbf{8 0} 249$

[43] Ferreira da Silva M F and Serrão J M P 2003 Journal of Physics B: Atomic, Molecular and Optical Physics 36 2357

[44] Oppenheimer J R 1928 Physical review 31349

[45] Eichler J K 1981 Physical Review A 23498

[46] Reinhold C, and Miraglia J 1987 Journal of Physics B: Atomic and Molecular Physics 20541

[47] Shakeshaft R 1974 Journal of Physics B: Atomic and Molecular Physics $\mathbf{7} 1059$ 